\title{
Successful radical resection of a leiomyosarcoma of the pulmonary trunk
}

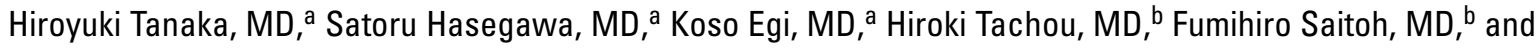 \\ Makoto Sunamori, MD, ${ }^{a}$ Tokyo, Japan
}

$\mathrm{P}$ rimary leiomyosarcoma of the pulmonary trunk is an extremely uncommon and highly lethal disease. The median survival without surgical resection is 1.5 months. ${ }^{1}$ Resection lengthens median survival to 10 months, and a few cases of long-term survival have been reported. ${ }^{1-5}$ We report a case in which a huge leiomyosarcoma of the pulmonary trunk was radically resected and reconstructed with the aid of cardiopulmonary bypass.

\section{Clinical Summary}

A 53-year-old woman was hospitalized for shortness of breath and a previous episode of syncope. Echocardiography showed a moderate amount of pericardial effusion and a large mass overlying the right and left ventricles. A sample of the pericardial effusion was negative for malignant cells. Computed tomography and magnetic resonance imaging disclosed luminal stenosis and a solid mass in the pulmonary trunk extending to the anterolateral surface of the heart (Figure 1, $A$ to $C$ ). Percutaneous needle biopsy from the anterior chest wall was performed. Microscopic examination and immunohistochemical staining led to a diagnosis of leiomyosarcoma. A right ventriculogram showed a mobile intraluminal mass and severe stenosis in the main pulmonary trunk (Figure 1, D). Right heart catheterization showed a mean right atrial pressure of $20 \mathrm{~mm} \mathrm{Hg}$ and a right ventricular pressure of 90/18 $\mathrm{mm} \mathrm{Hg}$. Coronary angiography showed compression of the left anterior descending coronary artery (LAD) and first diagonal artery, as well as a feeding artery originated the from LAD (Figure 1,E). Because of the absence of distal metastasis and intracirculatory tumor growth, she was transferred to our hospital for the radical tumor resection immediately after the diagnosis was confirmed.

The pericardium was opened via a median sternotomy. A huge mass overlying the pulmonary trunk and the anterolateral surface

\footnotetext{
From the Department of Thoracic and Cardiovascular Surgery, Tokyo Medical and Dental University, ${ }^{\mathrm{a}}$ and the Department of Cardiology, Tokyo Kensei Hospital, ${ }^{\mathrm{b}}$ Tokyo, Japan.

Received for publication March 20, 2001; accepted for publication March 27, 2001.

Address for reprints: Hiroyuki Tanaka, MD, Department of Thoracic and Cardiovascular Surgery, Tokyo Medical and Dental University, 1-5-45 Yushima, Bunkyo-ku, Tokyo 113-0034, Japan (E-mail: hiroyukitanaka.tsrg@ @md.ac.jp).

J Thorac Cardiovasc Surg 2001;122:1039-40

Copyright () 2001 by The American Association for Thoracic Surgery

0022-5223/2001 \$35.00+ $0 \quad \mathbf{1 2 / 5 4 / 1 1 6 1 9 6}$

doi:10.1067/mtc.2001.116196
}

of the heart was carefully dissected from the heart during ventricular fibrillation after cardiopulmonary bypass (Figure 2, A). The LAD and first diagonal artery were resected with the epicardial layer because of tight adherence of the mass to the epicardium. After careful dissection, most of the myocardium appeared free of tumor. Under cold cardioplegic arrest, the distal pulmonary trunk was transected, and intraluminal tumor was recognized (Figure 2, $B)$. The pulmonary trunk with the pulmonary valve and anulus and the right ventricular outflow tract including the myocardium of the interventricular septum, all invaded by the tumor, were resected en bloc with the already dissected huge mass. The resected area between the transected right ventricular outflow tract and the bifurcation of the pulmonary trunk was reconstructed with a 23-mm Carpenter-Edwards valved conduit (Baxter Healthcare Corp, Edwards Division, Santa Ana, Calif).

Pathologic examination of the solid white $15 \times 10 \times 10 \mathrm{~cm}$ tumor weighing $600 \mathrm{~g}$ (Figure 2, C) confirmed the diagnosis of leiomyosarcoma. Spindle cells with high mitotic activity, that is, mitotic counts of greater than 10 in 10 high-power fields, were observed. It appeared that the tumor originated from the posterior anulus of the pulmonary valve and then invaded the interventricular septum.

The patient was weaned from cardiopulmonary bypass with intra-aortic balloon pumping because of expected perioperative myocardial infarction caused by resection of the LAD and first diagonal artery. The early postoperative course was surprisingly uneventful, there was no perioperative infarction (maximum creatine kinase concentration, $1200 \mathrm{IU} / \mathrm{L}$; maximum creatine kinase MB concentration; $30 \mathrm{IU} / \mathrm{L}$ ), and the patient was discharged on postoperative day 30. No adjuvant radiation or chemotherapy was performed. To date, 36 months after the operation, the patient is doing well with almost no limitation in her daily activities and without evidence of recurrence.

\section{Comment}

Pulmonary trunk sarcoma is a rare and usually fatal disease. Radical surgical resection provides the only hope of long-term survival. ${ }^{1-5}$ Most primary leiomyosarcomas of the pulmonary trunk are associated with prominent endoluminal growth. The mass in this case, the largest we know of reported to date, was growing into the pericardial space as well as endoluminally, and it had invaded the pulmonary anulus, the interventricular septum, and the free wall of the right ventricular outflow tract. The epicardial layer, including the LAD and first diagonal artery, had to be excised so that the mass could be dissected from the heart. Coronary revascularization was not performed because the remaining distal segment of the LAD was too small. We expected perioperative infarction, but neither it nor pump failure occurred. 

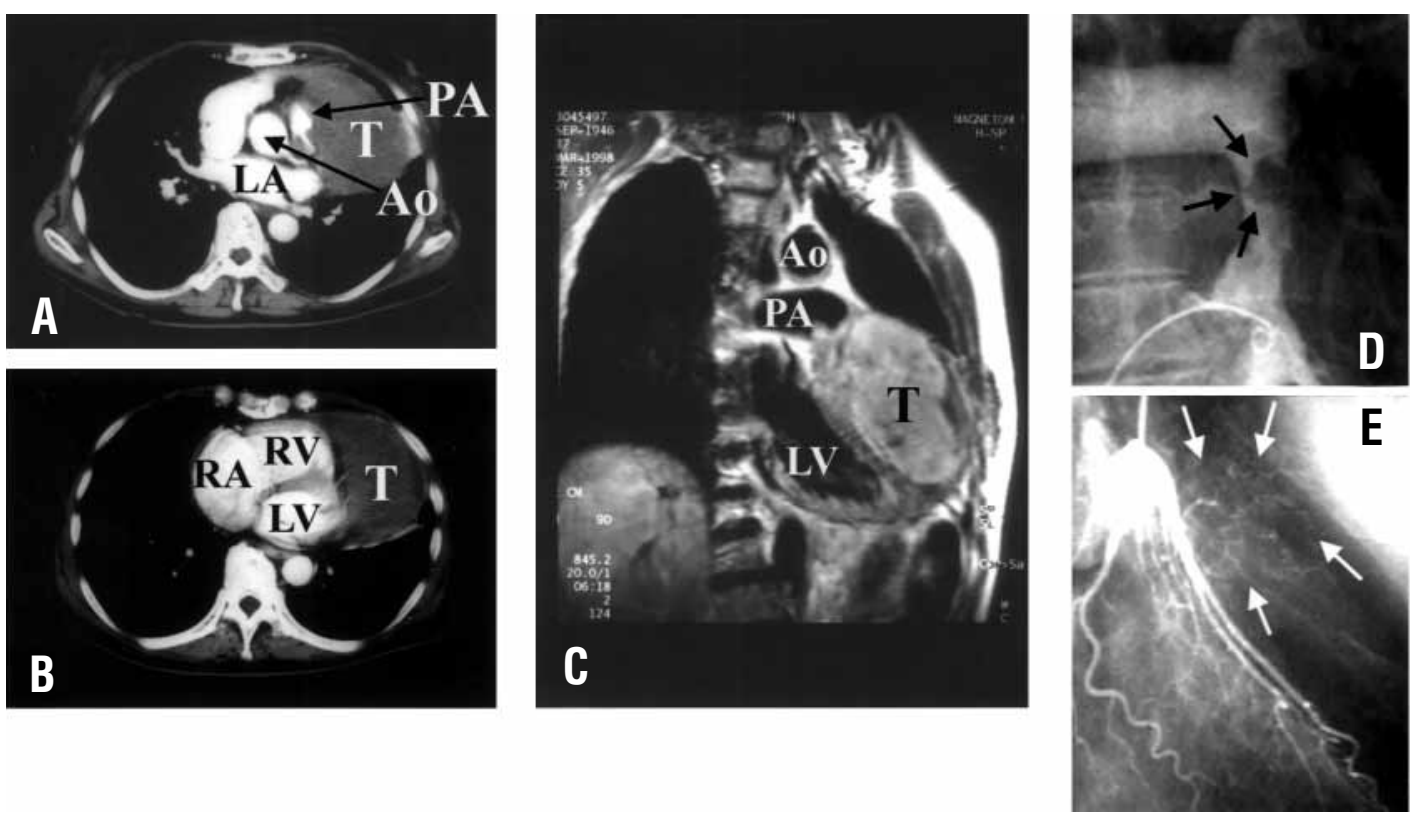

Figure 1. Preoperative computed tomographic scans (A and B) and magnetic resonance image (C) show the huge tumor growing within the pulmonary trunk and into the pericardial space. Right ventriculogram (D) shows a mobile intraluminal mass (black arrows). Left coronary angiogram (E) reveals a feeding artery originating from the LAD (white arrows). Ao, Aorta; $P A$, pulmonary artery; $L V$, left ventricle; $R A$, right atrium; $R V$, right ventricle; $T$, tumor.
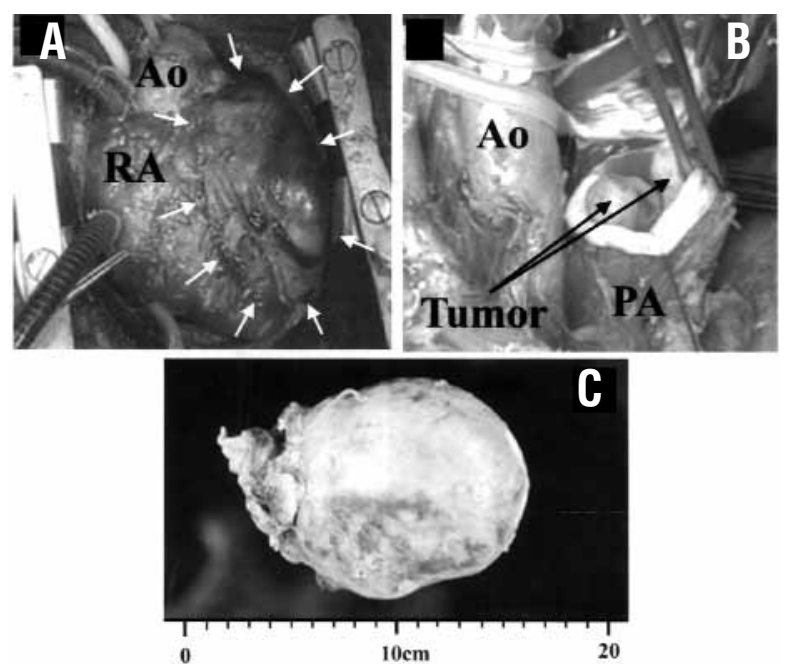

Figure 2. A, Intraoperative view after pericardiotomy showing a huge tumor overlying the anterolateral wall of the heart (arrows). B, Intraoperative view showing a lobulated tumor within the pulmonary trunk (arrows) after transection of the distal end of the pulmonary trunk. C, Anterior aspect of an excised specimen. Ao, Aorta; $P A$, pulmonary artery; $R A$, right atrium.
The postoperative left ventriculogram showed almost normal contraction with mild hypokinesis of the anterior wall, and the anterior septum was well perfused via the septal perforator from the posterior descending artery.

The role of adjuvant chemotherapy and radiation therapy is still controversial. ${ }^{2,3}$ Our patient refused both. The patient has survived 36 months with no sign of recurrence. Aggressive and extensive surgical treatment may provide significant palliation and opportunity for prolonged survival in other similar cases.

\section{References}

1. Kruger I, Borowski A, Horst M, de Vivie ER, Theissen P, GrossFengels W. Symptoms, diagnosis, and therapy of pulmonary sarcomas of the pulmonary artery. Thorac Cardiovasc Surg. 1990;38:91-5.

2. Mayer E, Kriegsmann J, Gaumann A, Kauczor HU, Dahm M, Hake $\mathrm{U}$, et al. Surgical treatment of pulmonary artery sarcoma. $J$ Thorac Cardiovasc Surg. 2001;121:77-82.

3. Zerkowski HR, Hofmann HS, Gybels I, Knolle J. Primary sarcoma of pulmonary artery and valve: multimodality chemotherapy and homograft replacement. J Thorac Cardiovasc Surg. 1996;112:1122-4.

4. Dossche K, Wellens E, Goldstein JP, Deferm H. Pulmonary homograft replacement for primary leiomyosarcoma of the pulmonary artery. $J$ Thorac Cardiovasc Surg. 1992;104:844-6.

5. Mazzucco A, Luciani GB, Bertolini P, Faggian G, Morando G, Ghimenton C. Primary leiomyosarcoma of the pulmonary artery: diagnostic and surgical implications. Ann Thorac Surg. 1994;57:222-5. 